\title{
Imaging Granulomatous Lesions with Optical Coherence Tomography
}

\author{
Christina Banzhaf Gregor B.E. Jemec \\ Department of Dermatology, Roskilde Hospital, Health Sciences Faculty, \\ University of Copenhagen, Copenhagen, Denmark
}

\section{Key Words}

Granuloma · Gout · Optical coherence tomography · Diagnostic imaging

\begin{abstract}
Aim: To investigate and compare the presentation of granulomatous lesions in optical coherence tomography (OCT) images and compare this to previous studies of nonmelanoma skin tumors.
\end{abstract}

Methods: Two patients with granulomas, tophi and granuloma annulare (GA), respectively, were photographed digitally, OCT-scanned and biopsied in the said order. Normal skin was OCT-scanned for comparison, but not biopsied. The OCT images from each lesion were compared with their histologic images as well as with OCT images with similar characteristics obtained from nonmelanoma skin tumors.

Results: The OCT images of the tophi showed hyperreflective, rounded cloud-like structures in dermis, their upper part sharply delineated by a hyporeflective fringe. The deeper areas appeared blurred. The crystalline structures were delineated by a hyporeflective fringe. OCT images of GA showed two different structures in dermis: a hyporeflective rounded one, and one that was lobulated and wing-like.

Conclusion: Granulomatous tissue surrounding urate deposits appeared as a clear hyporeflective fringe surrounding a light, hyperreflective area. The urate crystals appeared as hyperreflective areas, shielding the deeper part of dermis, meaning OCT could only visualize the upper part of the lesions. The lobulated, wing-like structure in GA may resemble diffuse GA or a dense lymphocytic infiltrate as seen on histology. The rounded structure in GA may represent an actual granuloma or either diffuse GA or a dense lymphocytic infiltrate as described above. This case suggests that OCT images granulomatous tissue as absorbent, hyporeflective areas, and urate crystals appear as reflective areas, obscuring the underlying tissue. In GA a new image shape looking like a wing has been found. The frequency, specificity and sensitivity of this new pattern in OCT imaging will require further studies. 
Optical imaging can be a helpful tool in the clinical management of many diseases. Optical coherence tomography (OCT) provides images with a resolution of $<7.5 \mu \mathrm{m}$ laterally, and $<10 \mu \mathrm{m}$ vertically. In terms of resolution this positions the method between high-frequency ultrasound and in vivo confocal microscopy.

OCT images display the classical layered architecture of the skin and may be used to identify keratinocytic tumors, especially basal cell carcinoma (BCC) and actinic keratosis. The details evident in the OCT images are, however, limited, and recognition is therefore based on an overall pattern recognition, rather than identification of finite elements such as e.g. individual cells. There is therefore a need to develop the understanding of the clinical correlate of OCT images, in order to optimize pattern recognition and the clinical utility of OCT imaging.

BCCs are seen in OCT as hyporeflective rounded structures from the epidermis extending into the dermis [1]. It is thought that the cell density of the tissue is an important factor in producing the OCT image because of the increased scatter due to the increased number of reflective elements in a more cellular tissue. It has also been suggested that images may appear blurred in cases where strong inflammatory infiltrate is found on histology [2].

Granulomas often present as localized and lobulated areas in dermis with a high cell density, and in e.g. granuloma annulare (GA), palisading of cells not unlike that seen in some BCCs is a common phenomenon (lymphocytes, histiocytes and fibroblasts surrounding a central degenerate collagen core). The aim of this study was therefore to describe the presentation of two types of granuloma in OCT images in order to explore the characteristics of these lesions.

\section{Materials and Methods}

OCT is an optical method which creates high-resolution images of e.g. skin through the use of infrared light and interferometry [4]. The OCT system used (VivoSight, Michelson Diagnostics, UK) is a multi-beam system, with a resolution of $<7.5 \mu \mathrm{m}$ lateral, and $<10 \mu \mathrm{m}$ vertical. OCT can image a cross section of the skin to the depth of 1-2 $\mathrm{mm}$.

Two granulomatous lesions were studied: tophi and GA. Tophi are histologically characterized by uric acid depositions in the dermis surrounded by granuloma formation with giant cells. Often, either occluded or dilated vessels are described. In polarized light the crystals appear sparkling [3].

GA is characterized histologically according to its clinical subsets. Common for all types is granuloma formation and degenerate collagen, often surrounded by a radially arranged infiltrate of lymphocytes, fibroblasts and palisading histiocytes. Among the subsets are diffuse and papular/generalized GA. Generalized GA microscopically resembles the above, while diffuse GA is characterized by collagen bundles arranged haphazardly with a circumferential lymphocytic infiltrate [3].

We aimed to visualize biopsy-verified granulomas with and without crystals to obtain a clear reference structure. After both patients provided informed consent, they were photographed digitally, OCT images were obtained both from lesional as well as normal skin, and biopsied in this order. The OCT probe was placed exactly on the lesions to be biopsied, and normal skin from the same body area was also OCT-imaged but not biopsied. 


\section{Case Presentation}

Case 1

A 50-year-old kidney transplanted male, presented to the Department of Dermatology of Roskilde with a 5-8 year history of white papules on the pulpa of the right phalanx of the 2nd finger (fig. 1a). The area had gradually expanded and measured $1.5 \times 1 \mathrm{~cm}$ and the elements $1-2 \mathrm{~mm}$. The elements were soft.

The lesions appeared as hyperreflective, rounded, cloud-like areas in OCT, their upper part sharply delineated by a hyporeflective fringe. The deeper areas appeared blurred. The length of the deposits was between 0.4 and $1.5 \mathrm{~mm}$, and OCT could visualize lesions to a depth of $1.5 \mathrm{~mm}$. The surrounding tissue appeared hyperreflective like the lesions, but was delineated because of the hyporeflective fringe (fig. 1b).

A lesional biopsy showed multiple uric acid depositions in the dermis with the presence of surrounding giant cells. The vessels appeared dilated (fig. 1c).

Case 2

A 61-year-old male was treated for GA. Clinically the patient presented papular/generalised GA. The papules were seen on both elbows (fig. 2a).

Lesional OCT scan showed changes in dermis consisting of hyporeflective, rounded and lobulated, wing-like structures found in the papular lesions of the elbows (fig. 2b). The structures measured between 0.03 and $2 \mathrm{~mm}$. The tissue above seemed partly compressed and a bulge equivalent to the lesion appeared in the epidermis. The vessels seemed dilated and more pronounced compared to normal skin, and the lobulated structure appeared in extension of a vessel (fig. 2b2).

Biopsy showed a chronic perivascular inflammation infiltrate and granulomas with necrobiosis and macrophages. Pronounced palisading was not seen. Central mucin deposits were found consistent with the diagnosis of GA (fig. 2c).

\section{Discussion}

Noninvasive diagnostics has numerous advantages for the patient as it may obviate the need for biopsies while enhancing the diagnostic accuracy. This is of particular relevance for medically treated conditions or research where longitudinal studies of lesions are of particular interest.

The cell density in the tissue plays an important role for the light scattering in OCT. In principle, not unlike granulomas, BCCs are cell-dense, often nodular or rounded structures, consisting of basal cells sometimes surrounded by inflamed tissue. It seems that BCCs present differently in OCT depending on the histological subset [5]. For example the BCC islands have been described as rounded hyporeflective structures sometimes surrounded by a hyperreflective structure thought to represent the stroma [1]. If inflammation or palisading is pronounced the image may appear blurred [2]. Granuloma may therefore be expected to have similarities with BCCs in OCT.

We studied two typical granulomatous structures: tophi and GA. In OCT imaging granulomatous tissue surrounding urate deposits appeared as a clear hyporeflective fringe surrounding a light, hyperreflective area (fig. 1b). The urate crystals appeared as a hyperreflective area, shielding the deeper part of dermis. OCT therefore only visualized the superficial part of the lesions. Vessels in images with uric acid depositions appeared more dilated compared to normal skin, probably because of the inflammation or a mechanical obstruction of the flow due to the deposit. The clear image of the urate crystals may be expected to be diagnostic for this condition. 
OCT imaging of GA identified two different images. One set of images (fig. 2b1) revealed rounded, hyporeflective, slightly blurred areas that did not appear specific. No associated stroma was seen. The other set of images (fig. 2b2) appeared more specific, resembling a bird wing. It consisted of a confluent series of rounded unreflective areas joined in a wing-like and lobulated oblong structure. This structure appeared very clear and hyporeflective. Vessels presented as dilated, and the structure appeared in extension of structures that could be interpreted as dilated vessels. The long lobulated OCT-imaged structure may represent an area of dense lymphocytic infiltrate as seen on histology. Alternatively it may represent diffuse GA, although the diffuse granuloma in the biopsy did not look as lobulated as the structure in the OCT image.

These cases suggest that OCT images granulomatous tissue as absorbent, hyporeflective areas, and urate crystals appear as reflective areas, obscuring the underlying tissue. In GA a new image shape looking like a wing has been found, which may either correspond with primary granulomas or the surrounding lymphocytic infiltrate. The frequency, specificity and sensitivity of this new pattern in OCT imaging will require further study.

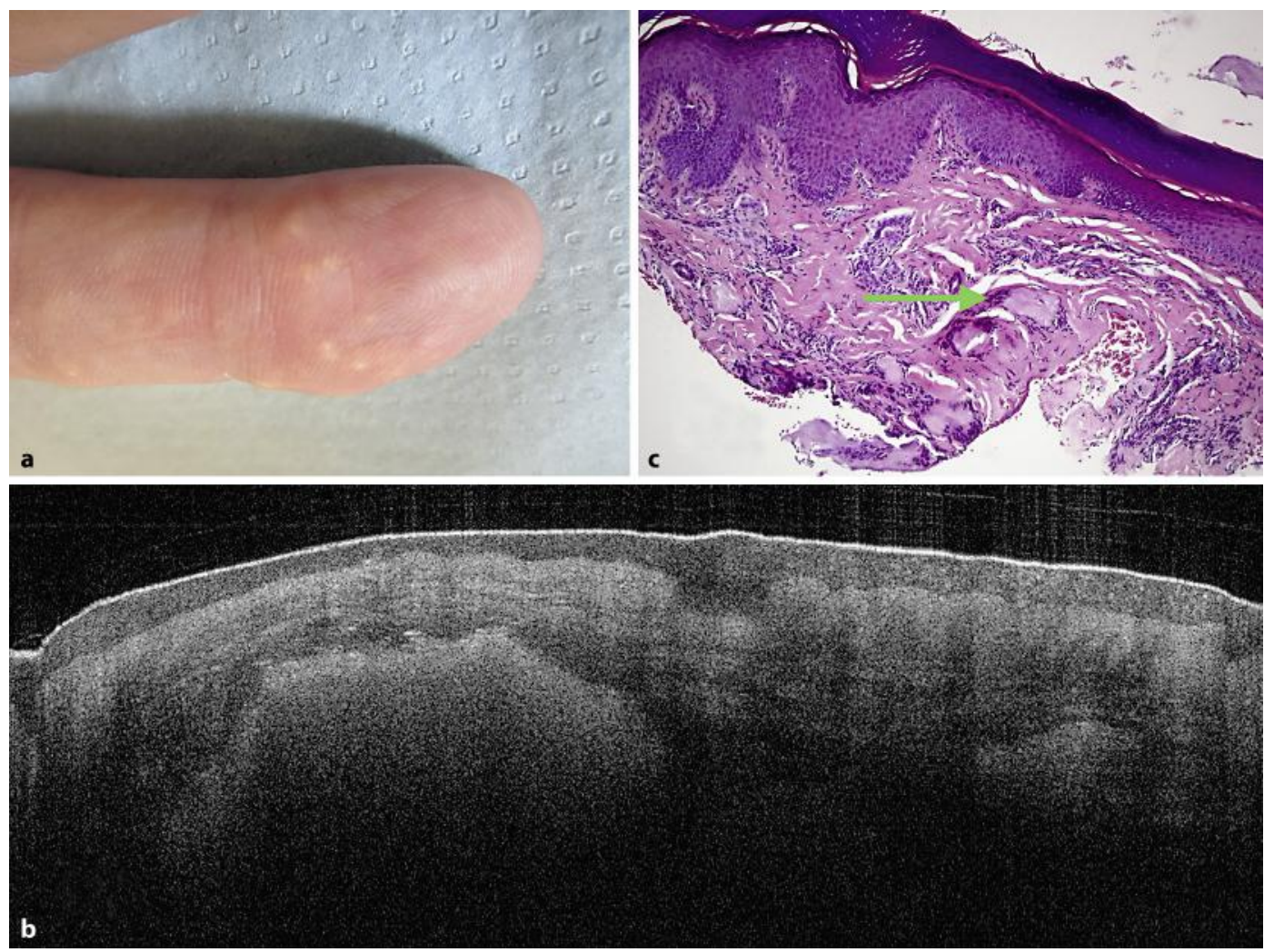

Fig. 1. a Tophi on the pulpa of the 2nd finger on the right hand. b Urate crystals in OCT appear as hyperreflective, rounded, cloud-like areas, the upper part sharply delineated by a hyporeflective fringe representing granulomatous tissue. c Hematoxylin-eosin stain of biopsy showing multiple uric acid crystals in the dermis with surrounding granulomatous tissue. 


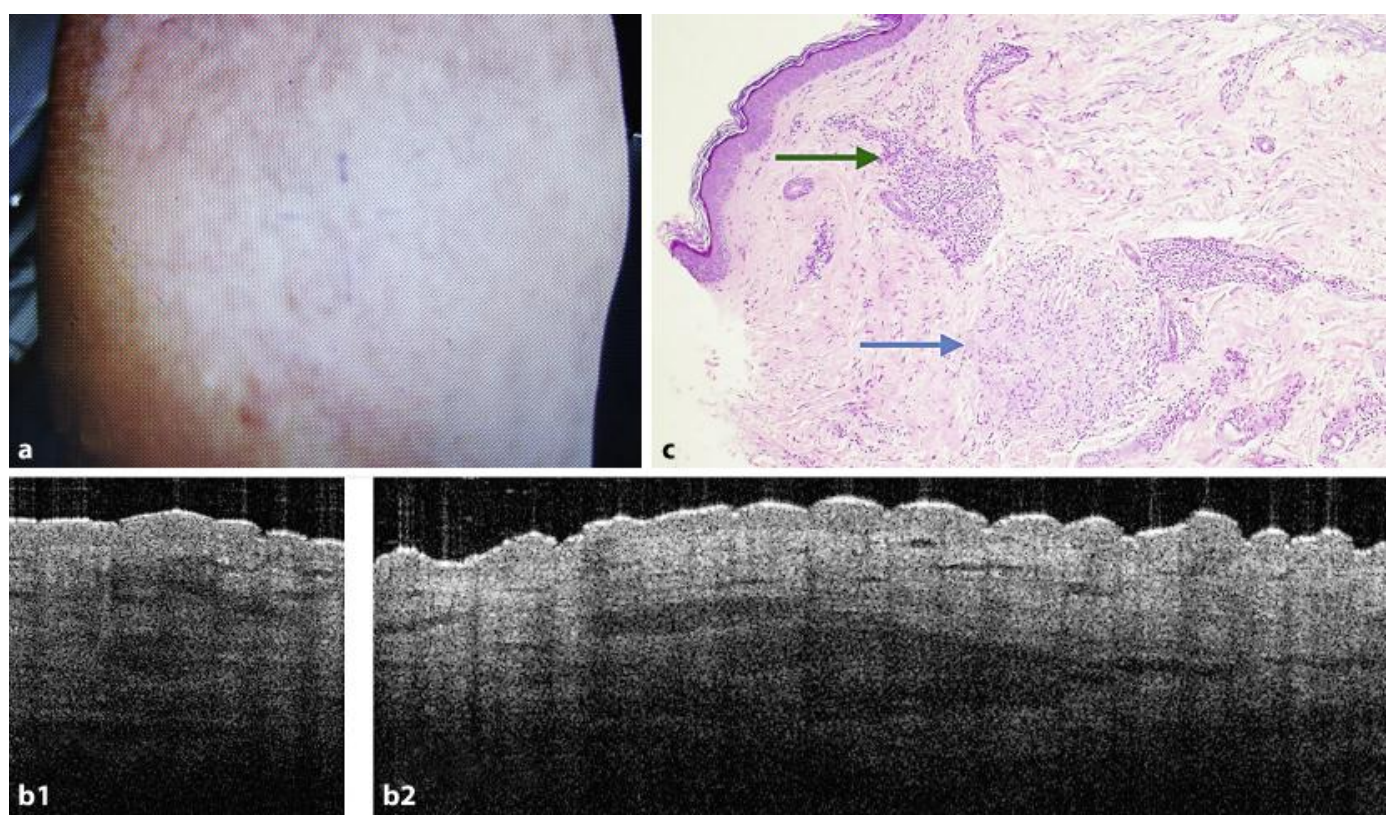

Fig. 2. a Papular/generalized GA on the right elbow of the patient. b1 OCT image of a rounded, nonspecific structure found in GA. Note the absence of the distinctive hyporeflective halo associated with basal cell carcinoma. b2 OCT image showing an oblong, wing-like structure that resembles the histological distribution of diffuse GA or a lymphocytic infiltrate. c Hematoxylin-eosin stain of biopsy of a GA papule showing a granuloma (blue arrow) and perivascular lymphocytic infiltrates (green arrow).

\section{References}

1 Mogensen M, Nürnberg BM, Forman JL, Thomsen JB, Thrane L, Jemec GBE: In vivo thickness measurement of basal cell carcinoma and actinic keratosis with optical coherence tomography and 20 MHz ultrasound. Br J Dermatol 2009;160:1026-1033.

-2 Mogensen M, Nürnberg BM, Thrane L, Jørgensen TM, Andersen PE, Jemec GB: How histological features of basal cell carcinomas influence image quality in optical coherence tomography. J Biophotonics 2011;4:544-551.

3 McKee PH, Calonje E, Granter SR: Pathology of the Skin, with Clinical Correlations, ed 3. Elsevier Mosby, 2005, vol 1-2, pp 297-305, 590-592, 1167-1184.

-4 Fujimoto JG: Optical coherence tomography for ultrahigh resolution in vivo imaging. Nat Biotechnol 2003;21:1361-1367.

5 Olmedo JM, Warschaw KE, Schmitt JM, Swanson DL: Optical coherence tomography for the characterization of basal cell carcinoma in vivo: a pilot study. J Am Acad Dermatol 2006;55:408-412. 


\section{Erratum}

In the article by Banzhaf C, Jemec GB: Imaging Granulomatous Lesions with Optical Coherence Tomography. Case Rep Dermatol 2012;4:14-18 (DOI: 10.1159/000336000), the following disclosure statement needs to be added:

Michelson Diagnostics provided the OCT system and technical support for this study. 